\title{
A test system for advanced lead acid battery state-of-charge and state-of-health research
}

\author{
Christopher R. Lashway, Peter Idowu \\ Pennsylvania State - Harrisburg, 777. Harrisburg Pike, Middletown, PA 17057, USA
}

\begin{abstract}
This paper presents a unique battery testing and quantification system to conduct advanced studies on battery Stateof-Health (SoH) and State-of-Charge (SoC). Improvements to the lead acid battery requires an intimate understanding of factors contributing to reduction of lifespan and causes of inaccurate SoC measurements. To study these properties, a testing system is developed with an objective that is two-fold. First, battery behavior and precise SoC is studied through life-cycle tests from inception to analyze charge efficiency and voltage stability during charge and discharge cycles. Secondly, time and frequency domain analysis are conducted to provide insights into spectral harmonics governing electrochemistry. By administering three unique test pulses after each charging cycle, a window into quantifying battery SoH and its correlation with inaccurate SoC measurements can be identified. The test system provides an industrial tool for analyzing battery performance, as well as an educational tool for studying the utilization of energy storage.
\end{abstract}

Keywords: Battery testing, state-of-charge, state-of-health, lead acid, energy storage

\section{Introduction}

The lead acid battery is the oldest battery in the market today having extensive use in portable power systems and accounts for over $40 \%$ of the battery sales to date. Its dependability and relatively lower cost has kept it in the spotlight, but its drawbacks are widely acknowledged. Presently encompassing the largest sector of the battery industry, lead acid has been used extensively in portable power systems for vehicles as well as in stationary applications such as Uninterruptible Power Supply (UPS) systems [1]. Although the current shift has been moving the industry away from its usage in future Electric Vehicle (EV) applications, their advantages in material availability and cost still maintain their importance in the market. Through the use of a flexible, unique testing tool to evaluate the lifespan and performance, present methods to extract State-of-Charge (SoC) and State-of-Health $(\mathrm{SoH})$ could be improved and managed in a more efficient way. This could be achieved through the deployment of an advanced battery lifespan and frequency analysis tool, such as the one developed in this paper, to highlight and improve the present models in-use today.

The first lead acid oxide cell was in 1859 by French physicist Gaston Planté when the system included two coiled strips separated by a simple linen cloth [2]. This simple demonstration laid the groundwork on how lead-acid batteries are manufactured today. Success has been attributed to many factors including low manufacturing costs and widely available raw materials. These reasons, coupled with relatively long life span have made the lead acid battery an efficient electrochemical energy-storage system. Performance of lead-acid batteries has significantly improved since they were introduced. Better designs with purer materials and efficient manufacturing techniques have lengthened the lifespan of these cells. Not only has dependability increased, but so has the focus on factors which eventually degrade and diminish the cycle

\footnotetext{
* Manuscript received October 20, 2015; revised January 19, 2016.

Corresponding author. E-mail address: clash006@fiu.edu.

doi: 10.12720/sgce.5.1.41-55
} 
life. Research has helped produce a maintenance-free battery that can last for several years when charged and discharged properly. The electrochemical processes that occur inside the lead acid battery can be represented by equations (1) and (2):

$$
\begin{aligned}
& \mathrm{Pb}+\mathrm{H}_{2} \mathrm{SO}_{4} \leftrightarrow 2 \mathrm{PbSO}_{4}+2 \mathrm{H}^{+}+2 \mathrm{e}^{-} \\
& \mathrm{PbO}_{2}+\mathrm{H}_{2} \mathrm{SO}_{4}+\mathrm{H}^{+}+2 \mathrm{e}^{-} \leftrightarrow 2 \mathrm{PbSO}_{4}+2 \mathrm{H}_{2} \mathrm{O}
\end{aligned}
$$

During the discharge phase (1) lead $(\mathrm{Pb})$ and lead dioxide $\left(\mathrm{PbO}_{2}\right)$ are converted into lead sulfate $\left(\mathrm{PbSO}_{4}\right)$ [1]. In the charge phase (2) this process is merely reversed. The composition and density of the sulphuric acid $\left(\mathrm{H}_{2} \mathrm{SO}_{4}\right)$ corresponding to a cell voltage will vary based on the State-of-Charge (SoC) of the battery. At full charge, the density is roughly $40 \%$ by weight of sulfuric acid and will produce $2.15 \mathrm{~V}$. When the battery charge has depleted, the density of sulphuric acid drops to around $1 \%$, yielding a lower cell voltage of $1.98 \mathrm{~V}$. The concentration of the sulphuric acid drives the cell voltage modelled by the Nernst equation [3]:

$$
E=E^{0}-\frac{2.303 R T \ln Q}{n F}
$$

where $E^{0}$ is the equilibrium cell voltage of $1.92 \mathrm{~V}, R$ is the ideal gas constant, $T$ is the absolute temperature, and $n$ is the number of electrons involved in the reaction and $Q$ is the reaction coefficient which is directly proportional to the sulphuric acid concentration.

$\mathrm{SoC}$ is directly correlated to the chemistry, but SoH has a strong correlation as well. Later in the battery's lifespan, a SoC reading can be very different from what is typically expected due to electrochemical changes occurring inside the battery cells. As a battery is charged and discharged several times it experiences cycling drive degradation [4]. Higher currents and deeper discharges put a higher strain on the battery and impact the usage sulphuric acid concentration. In addition to reducing the battery $\mathrm{SoH}$, the capacity is decreased as well. Through the use of the advanced battery test system presented in this paper, battery lifespan, performance, and key features in aging can be quantified. This system can be utilized for both the electrical or power engineer and electrochemist alike and highlights the importance of sophisticated energy storage analysis tools for the emerging Smart Grid.

This paper is organized as follows: Section 2 discusses a variety of SoC models in use, Section 3 discusses SoH, Section 4 discusses the design, Section 5 discusses the test system program interface, and Section 6 concludes this work.

\section{State-of-Charge (SoC)}

Determining the SoC of a lead acid battery is critical in assessing battery performance; therefore a SoC indicator serves as a "fuel gage" which determines the energy remaining in a battery before it has been fully depleted. For as long as rechargeable batteries have existed, so have SoC indicators, with earlier versions basing SoC indicators on battery cell voltages. These methods were originally used simply to protect the battery against overcharge or discharging below normal operating limits [5].

\subsection{Voltage-Based measurements}

The first attempt on a SoC indicator focused on the cell voltage drop caused by the varying density of sulphuric acid, with many versions surfacing in the 1960's. Curtis instruments pioneered gauges for monitoring the SoC of vehicle traction batteries and their reputation was world renowned with their SoC gauges selected for use in the Apollo spacecraft missions. Use of voltage-based estimation began to decrease quickly after the 1970 s as it was identified that relying on voltage alone for determining SoC proved to be an inaccurate indication of the battery's capacity. Cell voltage can fluctuate due to many other factors including temperature, discharge rates, and battery age [2]. Measurements must also be done while the battery has been at rest to achieve reasonable level of precision. 
Although there were many proprietary modifications to SoC indicators by developer/manufacturers, the basic model for the indicator is shown in (4):

$$
\operatorname{SoC}_{V}(t)=\frac{V_{f c}-V_{b a t t}(t)}{V_{f c}-V_{d c}}
$$

where the voltage-based state-of-charge $S o C_{V}$ is simply determined by the voltage (loaded) under full charge $V_{f c}$ subtracted by the current voltage $V_{b a t t}(t)$ over the gap between full charge and discharge $V_{d c}$. Due to severe nonlinearities in the voltage under load, this algorithm is highly inefficient.

\subsection{Voltage-Based measurements}

The most common way to measure battery SoC is a technology that has been implemented in many devices used today. Current integration or coulomb-counting uses a timing component and integrates the current each second to determine the capacity which has been removed or replaced in the battery. By measuring the current flowing into and out of the battery, the $S o C_{C}$ is calculated through a comparison with the total rated capacity [6]. The algorithm is shown in (5):

$$
\operatorname{SoC}_{C}(t)=\operatorname{SoC}\left(t_{0}\right)-\frac{1}{C} \int_{0}^{t} I_{b a t t}(\tau) d \tau
$$

where $I_{\text {batt }}$ is the battery current, and $C$ is the nominal capacity. In order to use this algorithm effectively, the initial SoC must be known. This requirement is typically overcome by performing a complete charge and discharge.

Coulomb counting IC's for accomplishing this function have been developed in the past few decades and have become relatively inexpensive [7]. Some devices have attempted to improve precision of SoC measurements as well as minimize the power consumption, however, the algorithms still lack information on variations in the internal battery characteristics and are unable to give advance warning of a battery failure [8]. Historical information of the battery is critical in order to develop an accurate SoC reading once the battery has begun to age. Advanced coulomb counting methods typically combine this method with higher processing book-keeping systems, involving more than an instantaneous current flow algorithm [9]. The unpredictable behavior of batteries and users requires the implementation of an adaptive system which combines instantaneous current flow with information on the battery chemistry and history. A "smart" coulomb counter is able to log data based on each discharge. Battery life is contingent on the depth of discharge and number of cycles to which the battery has been exposed. Many algorithms are being developed with these information enhancements to compensate for battery life and behavior.

\subsection{Higher level algorithms}

Although coulomb counting is the most widespread method in use for SoC implementation, many other higher level methods have been tested. Until coulomb-counting, measurements on the battery had been limited to current and voltage. To use many higher level algorithms, a new set of parameters and measurements must be obtained in order to achieve precision. Recent fuzzy logic based learning systems have been tested in an attempt to remove the time-dependent nature seen in coulomb counting and use other parameters to dictate the age and health of the battery on-demand. In [10], a simple fuzzy logicbased learning system is developed for an electric vehicle application. This system learns the battery signature by automatically creating a new fuzzy rule base. Similar to current integration, in order for this system to function properly the user must know the initial SoC of the battery. An error function is then developed using these values and once battery cycles have begun, they can be fine-tuned. This model produced small error after few cycles during controlled test conditions, but noted that further developments were still necessary to compensate for incomplete charge cycles as well as idle times. 


\subsection{Impedance spectroscopy}

The technique in [10] brought in some new parameters as well as defined an error function, but physical inputs to the system still relied a great deal on voltage measurements and current flow. More advanced methods have followed involving fuzzy logic and neural networks with a final decision function to determine SoC values. To improve accuracy and provide for more input to these decision functions, recent attention has focused on battery impedance measurements. Through impedance spectroscopy, the electrochemical characteristics of a battery are evaluated by applying an AC potential at many varying frequencies [11]. Frequencies from $1 \mu \mathrm{Hz}$ to $100 \mathrm{kHz}$ are typically stepped up logarithmically. It has been demonstrated that by applying a wide range of different AC frequency pulses, a wide range of characteristics about the battery history can be revealed.

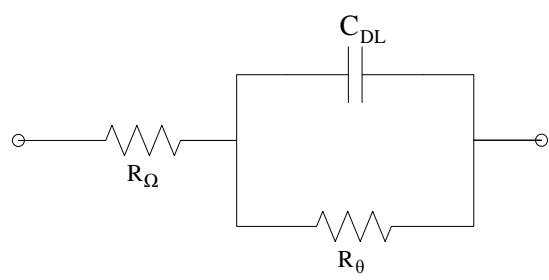

Fig. 1. Randles circuit [9].

To retrieve the electrochemical process information from the impedance spectroscopy results, a Randles circuit model is used for depicting each electrode. Shown above in Fig. $1, \mathrm{R}_{\Omega}$ represents the active electrolyte resistance and $R_{\theta}$ represents the charge-transfer resistance modelling the inherent speed of the chemical reaction. In a lead-acid battery, the internal electrolyte interface consists of several plates. This interface behaves like a capacitor and is referred to as double layer capacitance $\mathrm{C}_{\mathrm{DL}}$. The effective impedance for a single electrode $Z_{e}$ thus becomes nonlinear and is depicted by the Laplace transfer function:

$$
Z_{e}(s)=R_{\Omega}+\frac{R_{\theta} s^{0.5}+\sigma \sqrt{2}}{s^{1.5} R_{\theta} C_{D L}+s C_{D L} \sigma \sqrt{2}+s^{0.5}}
$$

where the electrochemical diffusion layer is added and depicted by $\sigma$ [12] .

In [12], Randles circuit is modified to include support for both battery terminals, doubling the parameters generated in the traditional design. An additional impedance value is also accounted for and is added in parallel with $\mathrm{C}_{\mathrm{DL}}$. These parameters provide for many new variables to build neural network training sets and fuzzy logic membership functions to help build a powerful tool for not only SoC, but a complete battery management system. The parameters obtained through this impedance model will generate values which can indicate battery age. With the proper equipment, impedance spectroscopy can be a very powerful tool in determining accurate battery SoC and SoH. It is important to note, however, that the AC frequency test cannot be conducted when the battery is under a load.

\section{State-of-Health (SoH)}

SoC is the most common assessment for a battery, but it is only the first step when looking at a total battery management system. Information over a battery's history is crucial to accurately calculate the SoC. Whether this history has been logged sequentially following each battery cycle, or impedance spectroscopy has modelled the chemical decomposition which has occurred over a battery's lifetime, information regarding the battery's condition is a critical factor [13]. The battery's condition, or SoH, is an assessment of how its performance will vary with age. Due to irreversible physical and chemical changes that take place with usage, ultimately a battery will reach a point where it can no longer source its rated current. The degradation and aging of a battery is a complex process that involves many 
parameters, most of which impact capacity. Over a battery's lifespan, the available capacity following a full charge will decrease.

$\mathrm{SoH}$ is an important trait to model as later in the battery's lifespan, the SoC reading can be very different from what is typically expected. Advanced book keeping systems feature a way to model how a battery's SoH will decrease over time, with nominal capacity ratings needed to model the SoH degradation over later cycles [14]. These specifications are typically provided within a battery's datasheet and dictate the capacity which can be achieved when different currents are applied. The capacities will show a downward trend as the battery begins to age. Standards have been developed to coordinate a discharge current with the amount of time it will take before the battery has fully depleted. These rates progress from a 20-hour discharge rate using a lower constant current level and step down to a 15-minute discharge under very high current. The 20 -hour discharge rate features a conservative load current in which the full capacity of the battery can be obtained when it is healthy.

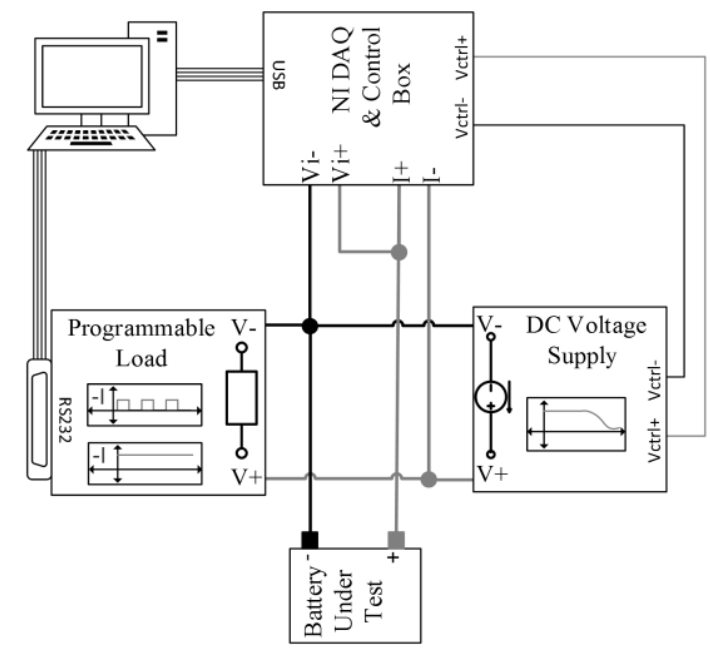

Fig. 2. Battery test system connection.

\section{Battery Test Stand Design}

In designing a test system for a battery, the designer needs to be extremely conscious of connections and cable lengths to reduce voltage drops and noise to ensure high fidelity measurements and an accurate frequency domain representation. The test system had to be capable of charging and discharging the battery while also providing support for a number of additional measurement devices and minimizing noise. There are four major components in the test system which are depicted in Fig. 2: Battery under test, a programmable load, a DC voltage supply, and a Data Acquisition system interface to the control software.

\subsection{Software data acquisition and control}

The LabVIEW Development Platform was selected to program the battery test stand software. LabVIEW is an excellent solution to control, obtain accurate measurements, and provide efficient logging of data for later processing. Testing of a lead-acid battery is a lengthy process with each cycle taking up to 13 hours or more. The required software must run for days and weeks at a time with little to no support from the user. In order to run the battery test stand on its own, each phase needed to be carefully set up in order to prevent the overflow of data or damage to the equipment. The basic model of the program needed to first query information about the battery being tested before initiating battery cycles. After initial parameters have been set, the system follows the flow diagram shown in Fig. 3. General phases include battery charging, applying of test pulses using an electronic load, and then finally, discharging the battery. 
This basic model does not include several other stages which are needed to control the equipment and simulate realistic battery treatment, they are however described in detail in the following sections. To protect the battery from premature damage, rest periods are included before and after every phase transition.

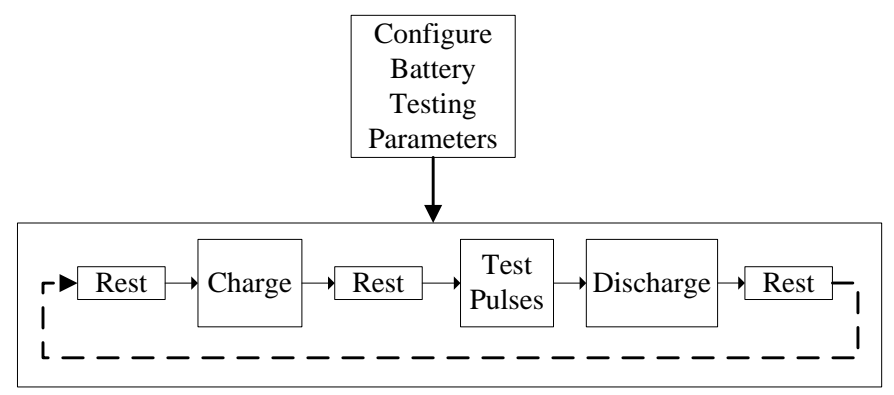

Fig. 3. Enhanced battery test stand flow diagram.

Rest periods initially default to 5 minutes but can be set to a value of 1 minute or higher by the user before the testing begins. Comparable to an oversized capacitor, a battery is known to have a very slow response to change and long periods are recommended [14]. Quick switching between charging, test pulses, and discharging phases can inadvertently damage the battery. Rest periods lead to more accurate results as well as preserve the battery's chemistry.

\subsection{Test configuration}

Upon launching the Battery Test Stand software, the first screen that is displayed is the "Test Configuration" screen which is depicted in Fig. 4. The primary purpose of this program is to query information from the user about how the battery is to be tested during each phase as well as set conditions for what should terminate the test. The "Configure a Test" screen is divided into three major sections: Test Parameters, Charging Setup, Discharge Setup, and the End-of-Test Condition.

The first column sets up basic test parameters and begins with a user selection over whether a battery test is being continued or started at its first cycle. When the user selects "Setup New Test," certain parameters are disabled in order to prevent initial values from being set. However, when "Continuing Test" is selected, five parameters are enabled for the user. The first parameter is the "Initial Cycle Number" which sets the last cycle number the battery completed. The four remaining parameters allow the user to input information about previous cycles into the system. Both the charge and discharge columns permit the user to input the total elapsed time and capacities which have been transferred since the first cycle. When a user has chosen to continue a test, referencing the data files from a previous test will provide these four values. As shown in Fig. 4, the previous cycle that the battery test had completed was 46. Upon referencing data taken in Cycle 46, it was found that as of this point the battery had charged a total of $18,190.8$ minutes and input 1,106.457 Ah into the battery. During this same period, the battery had discharged 10,343.1 minutes and output a total of $884.457 \mathrm{Ah}$. Three parameters that must always be set regardless of the type of test is the "Rest Period between Stages," the "Data Collection Interval," and the "Folder Location to Store Data."

Finally, the user must also designate how often the software should collect measurements as well as a location to save all test data. The data collection interval is set at default to be 1 minute. The user may set this to as low as 1 second, however, a 1 minute interval is recommended in order to control file sizes and prevent running down CPU processing time. The folder to store this and the rest of the test data must also be selected. Once a test has completed its first cycle, primary data taken at every collection interval, screenshots of each phase, voltage and current measurements during the test pulse period, and the final details over each cycle are saved with file names correlated to the time stamp, cycle number, and phase in which it was taken. 


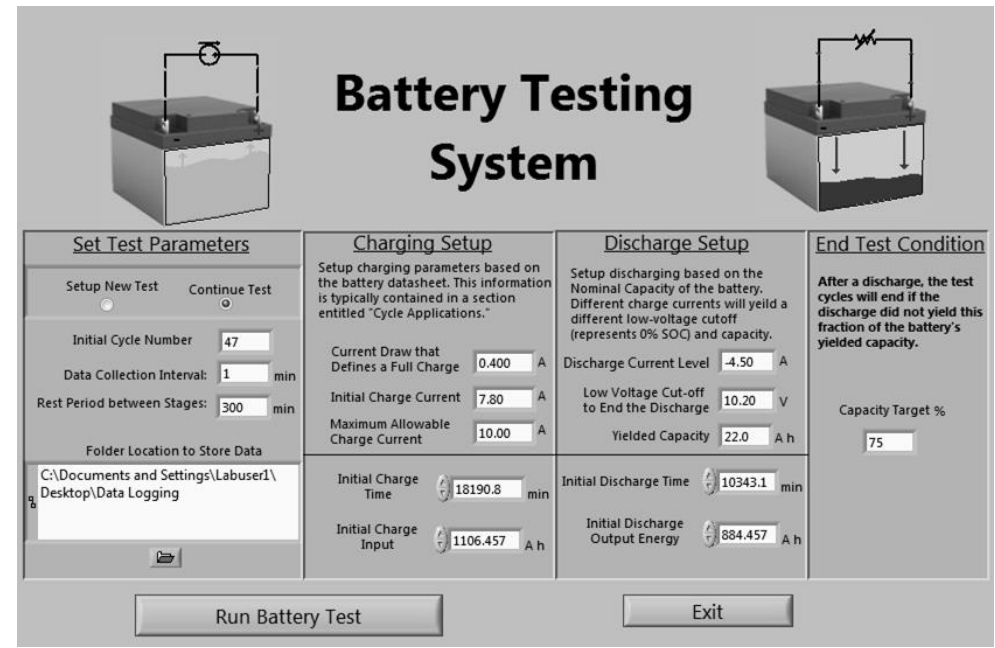

Fig. 4. Test configuration screen.

\subsection{Charging phase}

The following column in the test configuration sets up the charging parameters of the battery test. Charging a battery is a three-stage process which starts with "constant current," progressing to a "constant voltage," and ends with a "float charge" phase [2]. Parameters to program each of these stages are outlined in the battery datasheet [15]. The charging process is explained in-detail in following sections. A HP 6267B power supply was used in this test to charge the battery [17]. This power supply must have the voltage adjusted by the user before conducting a test as only current can be controlled by the software. There are several ways in which the HP 6267B can be controlled, though in this case, an analog voltage sourced from the DAQ was the most practical solution. Setting up this supply to be voltage-controlled was a relatively simple process involving 5 jumpers and one $130 \Omega$ resistor (Fig. 5). The HP 6267B manual outlined the current programming coefficients needed to control the power supply using an analog control voltage. For this particular model, the coefficient is $50 \mathrm{mV} / \mathrm{A}$ over a voltage range of 0-500 mV.

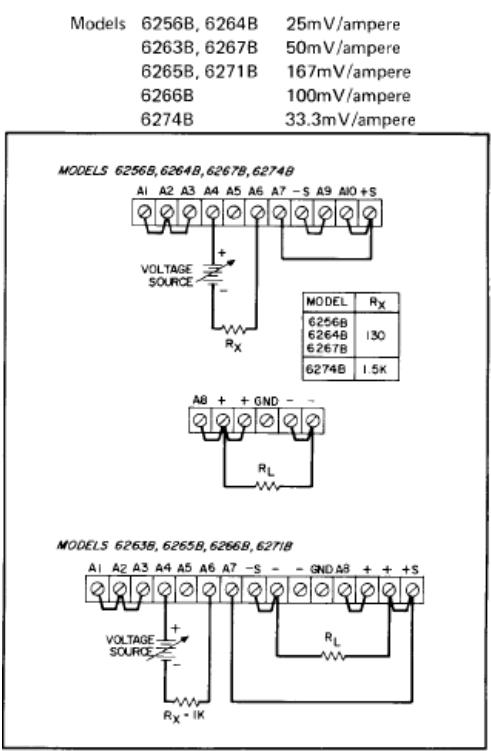

Fig. 5. HP 6267B voltage programming of output current. 
Beginning with the constant-current stage, the maximum current the equipment was able to source is used. The HP 6267B is able to source up to $10 \mathrm{~A}$, but for many batteries, an initial charging current of 10 A is too high. Before the charger is set to the maximum charging current, a smaller initial charge current is used as outlined in the datasheet [15]. For this test, the "Initial Charging Current," shown in Fig. 4, is 7.8 A. Using a timer function provided in LabVIEW, the battery charges at 7.8 A for the first 30 seconds, then proceeds to ramp the current to its maximum value of $10 \mathrm{~A}$. The LabVIEW block diagram for the charge control circuit is shown in Fig. 6. A timed loop is used in conjunction with a shift register to slowly ramp the current up to its maximum level. This sequence prevents the charger from instantaneously setting the current to its target and instead, gradually steps up the current over a 10 second period. There is also an added safety feature which will completely stop the charger if the battery voltage rises above $17 \mathrm{~V}$.

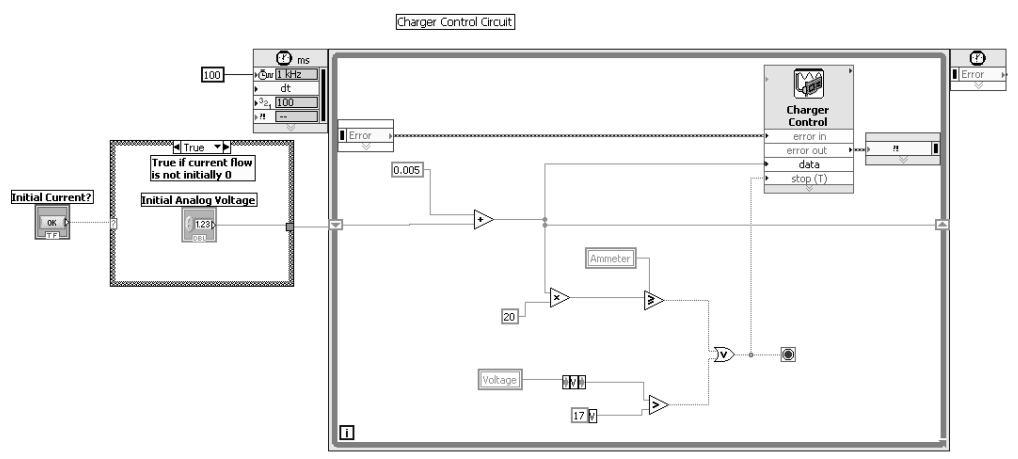

Fig. 6. Charger control algorithm.

Once the first stage of charging has completed, the voltage is held at a constant value as the level of current begins to decrease. This stage is an indication of the sulphuric acid concentration becoming saturated and tends to indicate that the battery has achieved around $60 \% \mathrm{SoC}$ [16]. Following this point, the charger will then remain at its maximum current and controlled voltage $(14.7 \mathrm{~V})$ levels, but the current entering the battery will begin to fall indicating it has entered "float charge." Ultimately, the battery will reach a threshold where the level of current entering the battery will no longer decrease indicating it has reached $100 \%$ SoC. This threshold is referred to as the "Current Draw that Defines a Full Charge" (in Fig. 4) and will instruct the software that the battery has completed the charging phase.

The battery charging behaviour can also provide insight into the battery SoH. First, monitoring the quantity of energy injected into the battery prior to the transition between the constant current and constant voltage phase can provide the operator with a reasonable expectation of the remaining usable capacity. This can be easily calculated using the expected capacity range which should be present around $60 \%$ SoC. Of course, this figure would be expected to drift from battery to battery but a large deviation would indicate a battery which has lost a great deal of its usable capacity. Secondly, monitoring the duration of the constant current phase with respect to the duration of the constant voltage and float charge periods can provide further SoH information as well. If the constant voltage and float charge periods last longer than that of the constant current period, this also provides a strong indication of a weak battery which has lost a great deal of its initial capacity.

\subsection{Test pulse phase}

An Amrel Programmable Electronic Load is used in order to administer all test pulses as well as discharge the battery [18]. Remote programming of the Amrel is done serially using RS-232 and a full list of commands and syntaxes are outlined in the Amrel Programming Manual. String-based instruction sets are transmitted to the Amrel device to program each test pulse. Three distinct pulses are run between every charge and discharge phase in the battery test. All pulses vary the discharge current and last for 5 seconds each. In order to allow time for the equipment to register each command, each test pulse 
instruction is sent with a $50 \mathrm{~ms}$ wait period. After this period has elapsed, the system proceeds to record the time domain and Fast Fourier Transform (FFT) of both the current and voltage responses.

The first test pulse selected is of sinusoidal form. Shown in Fig. 7, the Amrel instruction sets a starting current level of $6.0 \mathrm{~A}$ and peaks around 6.7 A. The instruction set goes on to set properties of a transient signal operating at a frequency of $133 \mathrm{~Hz}$. The second test pulse administers is a square wave. This square pulse was selected due to its recent success in advanced SoH devices and operates at a frequency of $1 \mathrm{kHz}$ [19]. The square wave has a much wider variation of the current level and is instructed using a $40 \%$ duty cycle. Each cycle holds the current at $10 \mathrm{~A}$ for $40 \%$ of the period and $5 \mathrm{~A}$ for $60 \%$ of the period. The instruction set and current response of this pulse is shown in Fig. 8.
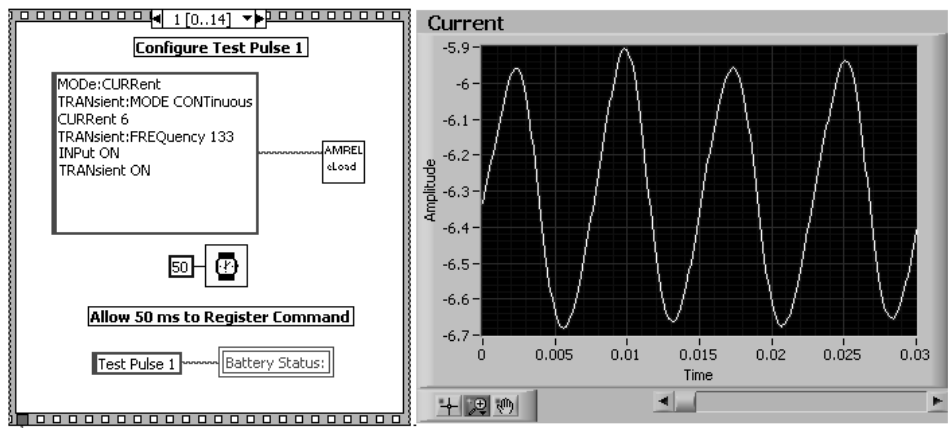

Fig. 7. Test pulse 1: Instruction set (left), current response (right).
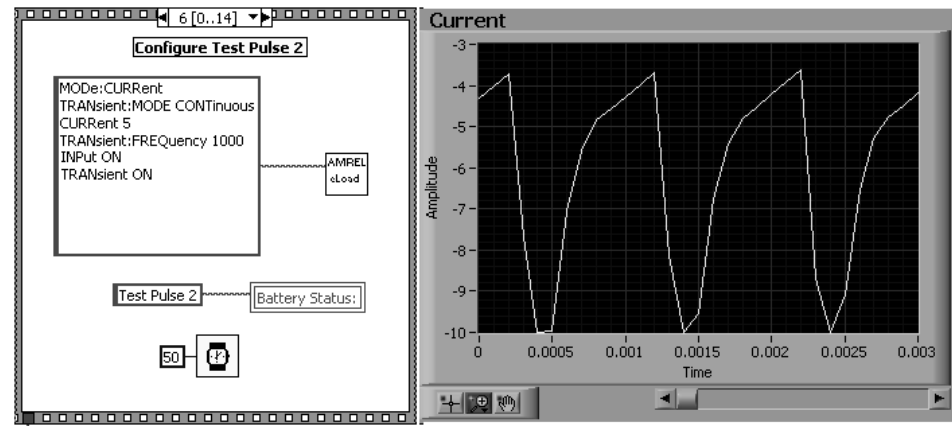

Fig. 8. Test pulse 2: Instruction set (left), current response (right).
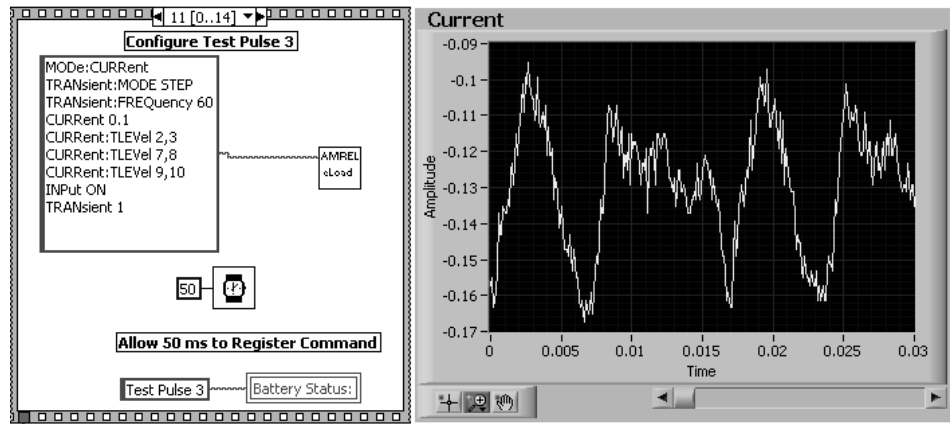

Fig. 9. Test pulse 3: Instruction set (left), current response (right).

The third and final test pulse introduces a stepping element to the battery. This pulse operates at a much lower level of current and was selected due to the unique frequency response it revealed on a fresh battery. Shown in Fig. 9, this pulse operates at a much lower frequency of $60 \mathrm{~Hz}$ and features a starting current level of only $100 \mathrm{~mA}$. This pulse features 5 different parts during one cycle. The first part sets the current to its maximum of $170 \mathrm{~mA}$. The second step drops the current to $110 \mathrm{~mA}$ and performs three 
additional steps where the current is increased by $10 \mathrm{~mA}$ per step. Following these three steps, the pulse cycle ends back at its maximum level of $170 \mathrm{~mA}$. Due to the small magnitude of current being drawn, a great deal of noise is noticeable when viewing the pulse in the time domain.

\subsection{Frequency domain analysis}

Although a time-domain analysis of the test pulse responses reveals the instantaneous battery response, a frequency domain power spectral analysis provides the user with much greater insight into battery impulse response and behaviour. Power spectral analysis is closely related to the Fast Fourier Transform (FFT) of the signal, which extracts the harmonic components of the impulse response [20]. The Fourier Transform of the voltage $V(f)$ and current $I(f)$ is:

$$
\begin{aligned}
& V(f)=F\{v(t)\}=\int_{-\infty}^{+\infty} v(t) e^{-j 2 \pi f t} d t \\
& I(f)=F\{i(t)\}=\int_{-\infty}^{+\infty} i(t) e^{-j 2 \pi f t} d t
\end{aligned}
$$

where $v(t)$ and $i(t)$ represent the time-domain voltage and current signals of the battery under test and $\mathrm{f}$ represents the frequency.

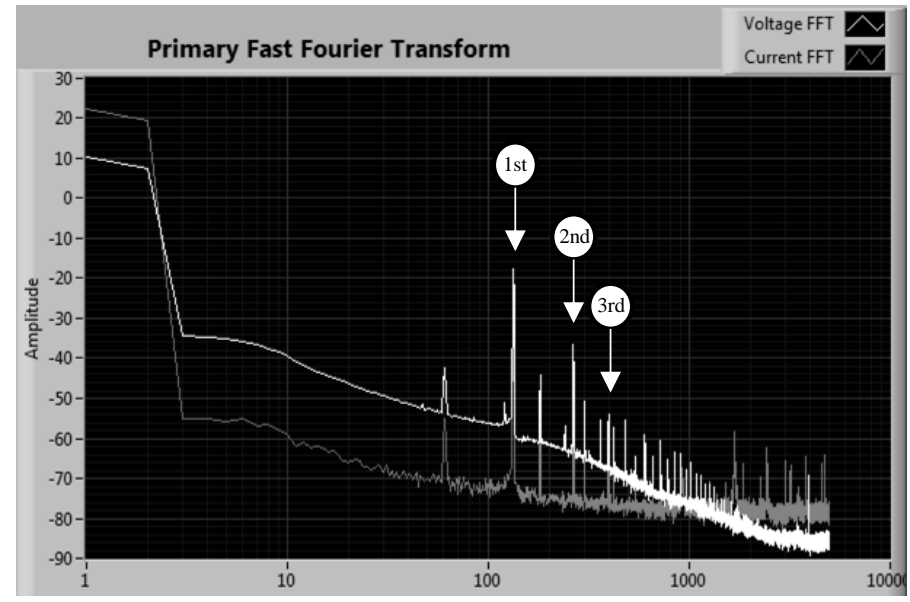

Fig. 10. DFT display of test pulse 1 in cycle 1.

The power spectrums $V_{x x}(f)$ and $I_{x x}(f)$ to calculate the harmonic content of the signal is calculated by the product of the FT multiplied by its complex conjugate.

$$
\begin{aligned}
& V_{x x}(f)=V(f) V^{*}(f)=|V(f)|^{2} \\
& I_{x x}(f)=I(f) I^{*}(f)=|I(f)|^{2}
\end{aligned}
$$

Since the Fast Fourier Transform (FFT) is represented as the discrete FT, the power spectrum is also interpreted in discrete time:

$$
\begin{aligned}
& V_{x x}=F\{v(t)\}^{2} n^{-2} \\
& I_{x x}=F\{i(t)\}^{2} n^{-2}
\end{aligned}
$$

where $n$ represents the number of elements passed inside the voltage and current vectors $\mathrm{v}$ and $\mathrm{i}$. For this system, 10,000 samples are gathered at a sampling rate of $10 \mathrm{kHz}$.

The DFT of the voltage (white) and current (grey) during Test Pulse I (133 Hz) reveal these characteristics and are shown in Fig. 10. The Nyquist criterion governs the frequency span to $10 \mathrm{kHz} / 2$ or 
a sweep to $5 \mathrm{kHz}$. The peak response is present at the fundamental, but there are prominent ripple magnitudes at the 2nd and 3rd multiples. Other frequency components are present outside of the standard orders which can help to pinpoint the lifetime and electrochemical properties of the battery. This tool can help to provide an advanced analysis into performing preliminary impedance spectroscopy measurements.

\subsection{Discharging phase}

The "Discharge Setup" column, similar to the "Charging Setup" column, requires information from the battery's datasheet [1]. This phase terminates once the battery voltage drops below a cut-off value coordinated its nominal capacity. Higher discharge currents in a cycle will yield lower capacities. In conjunction with a lower capacity, a lower cut-off voltage is also observed. The discharge level is programmed to the Amrel similar to the test pulses using an instruction set sent over RS-232 [18]. Shown in Fig. 11, a 5-line string-based instruction set is transmitted to the Amrel device to set the constantcurrent level.

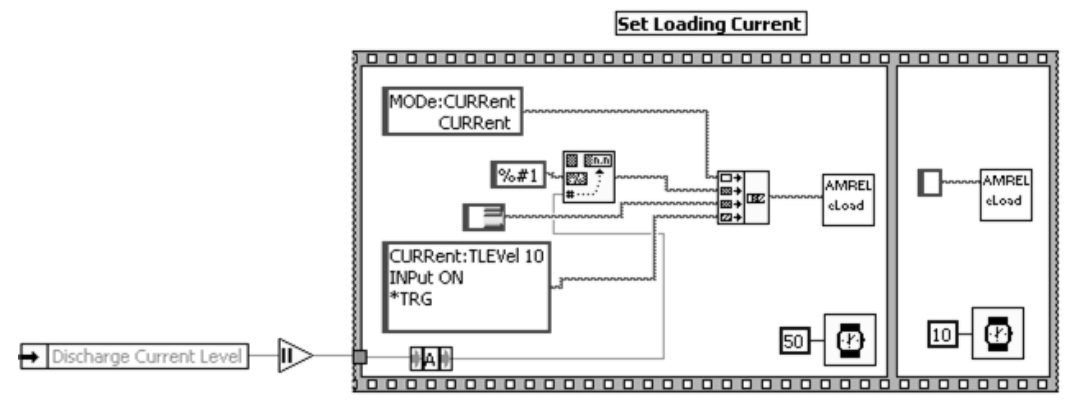

Fig. 11. Electronic load control algorithm.

Table 1. Nominal capacities for power sonic 26 Ah PS-12260.

\begin{tabular}{|r|r|r|r|}
\hline Discharge Rate & Discharge Current & Cut-off Voltage & Capacity \\
\hline 20-hour & $1.3 \mathrm{~A}$ & $10.50 \mathrm{~V}$ & $26.0 \mathrm{Ah}$ \\
\hline 10-hour & $2.4 \mathrm{~A}$ & $10.50 \mathrm{~V}$ & $24.0 \mathrm{Ah}$ \\
\hline 5-hour & $4.4 \mathrm{~A}$ & $10.20 \mathrm{~V}$ & $22.0 \mathrm{Ah}$ \\
\hline 1-hour & $16.1 \mathrm{~A}$ & $9.00 \mathrm{~V}$ & $16.1 \mathrm{Ah}$ \\
\hline 15-minute & $51.0 \mathrm{~A}$ & $9.00 \mathrm{~V}$ & $12.8 \mathrm{Ah}$ \\
\hline
\end{tabular}

The loading current is programmed using a 2-part timed sequence, operating from left to right. Similar to the test pulse instructions, a brief $50 \mathrm{~ms}$ pause allows time for the Amrel to register the command. Following the $50 \mathrm{~ms}$ wait, a blank transmission is sent to the device instructing the Amrel to hold its previous command until another instruction is sent. The discharge test relies on the battery voltage level to determine whether or not it has completed. A cut-off voltage for a full discharge is set for $10.5 \mathrm{~V}$ at the slowest (20-hour) discharge rate; however, it can fluctuate to as low as $9.0 \mathrm{~V}$ when higher currents are used as shown in Table 1. The user is able to set the discharge current level up to $10 \mathrm{~A}$. Table 1 shows a list of the nominal capacities associated with the Powersonic PS-12260. Nominal capacities are categorized by their rate of discharge. Five standardized discharge rates are available for this battery. In this test, a 5-hour discharge profile is used where the discharge current level is set to $4.5 \mathrm{~A}$. The associated cut-off voltage for this profile is lowered to $10.2 \mathrm{~V}$ where an expected capacity to be returned is $22 \mathrm{Ah}$ (Fig. 4).

The loading current is programmed using a 2-part timed sequence, operating from left to right. Similar to the test pulse instructions, a brief $50 \mathrm{~ms}$ pause allows time for the Amrel to register the command. Following the $50 \mathrm{~ms}$ wait, a blank transmission is sent to the device instructing the Amrel to hold its previous command until another instruction is sent. The discharge test relies on the battery voltage level to determine whether or not it has completed. A cut-off voltage for a full discharge is set for $10.5 \mathrm{~V}$ at the slowest (20-hour) discharge rate; however, it can fluctuate to as low as $9.0 \mathrm{~V}$ when higher currents are 
used as shown in Table 1. The user is able to set the discharge current level up to $10 \mathrm{~A}$. Table 1 shows a list of the nominal capacities associated with the Powersonic PS-12260. Nominal capacities are categorized by their rate of discharge. Five standardized discharge rates are available for this battery. In this test, a 5-hour discharge profile is used where the discharge current level is set to $4.5 \mathrm{~A}$. The associated cut-off voltage for this profile is lowered to $10.2 \mathrm{~V}$ where an expected capacity to be returned is $22 \mathrm{Ah}$ (Fig. 4).

\subsection{Cycle and test completion}

After all three phases have been completed, a final decision must be made to determine whether the battery should proceed into an additional cycle or end the test. The final column in the test configuration (Fig. 4) sets an expectation to the amount of capacity that the battery should be able to source before the test is ended. This value is calculated by a percentage of the "Yielded Capacity" value which was set in the "Discharge Setup" column. This value indicates the expected amount of energy that should be sourced when the battery is in a perfect SoH at the correlated discharge current. However, as the SoH of the battery decreases, the amount of energy it can source will decrease as well. In order to continue the battery test, the battery must be able to source a percentage of this capacity. In this case, the 22 Ah capacity is expected to source $75 \%$ of its rating (16.5 Ah). Once the battery completes a cycle where it sources less than $16.5 \mathrm{Ah}$, the test will end.

Table 2. Data collection file construction

\begin{tabular}{|ll|ll|}
\hline \multicolumn{2}{|c|}{ Primary Data Collection File } & \multicolumn{2}{|c|}{ Test Pulse Measurements } \\
\hline 1. & Voltage (V) & 1. & Voltage (V) \\
2. & Current (A) & 2. & Current (A) \\
3. & DFT Threshold (dB) & 3. & Voltage DFT (dB) \\
4. & Average Phase (degrees) & 4. & Current DFT (dB) \\
5. & Power (W) & & \\
6. & Charge Time (min) & & \\
7. & Charge Input (Ah) & & \\
8. & Cumulative Charge Time (min) & & \\
9. & Cumulative Charge Output (Ah) & \\
10. & Discharge Time (min) & \\
11. & Discharge Output (Ah) & \\
12. & Cumulative Discharge Time (min) & \\
13. & Cumulative Discharge Output (Ah) & \\
14. & Cycle Number \\
15. & Time Stamp \\
16. & Battery Status & \\
\hline
\end{tabular}

\subsection{Data logging}

There are three major categories of data logging which are present in each battery cycle. Of these three categories, two are geared toward the development of a precision SoC value to correlate with other equipment, and the final toward estimating the battery's SoH. Formatting is shown in Table 2.

Final SoC estimation has two different strings of data which can be processed following every battery cycle. Primary data collection extracts the most information about the battery and is observed at a 1minute interval over the entire cycle. These data files log the voltage, current, power, and capacities which were transferred during that cycle as well as cumulatively over the entire test. A full list of the measurements contained in this file is shown in Table 2. Nearly 500 samples are taken during each battery cycle providing enough to reconstruct a complete cycle profile. After each cycle has executed, a second data file is created with the same format of measurements except only one entry outlines the final capacities which have been charged and discharged from the battery as well as the duration of each phase.

The "Test Pulse Measurements" data files have a very different approach. The goal of these data files is to provide precise measurements of the battery's voltage and current while it is in the test pulse phase. Test pulses are administered for 6 seconds each in-between each charge and discharge cycle. Voltage, current, and the DFT of each are recorded into this file at 10,000 samples per second where an example of 
a real-time measurement response is shown in Fig. 12(b).

\section{Battery Test Stand Program Interface}

The battery test stand program interface is shown in Fig. 12 providing the user a great deal of information over the progress and current state of the test. Due to the large data sets available through the interface, all information with the exception of current "Battery Status" is organized into tabs where the user can select what information to view. The "Battery Status" not only indicates which phase and test pulse the battery is in but also warns the user when voltage and current levels reach unsafe levels. Two tab controls are featured inside the battery test program and separate the screen in two columns: one to display instantaneous measurements and another to toggle between the time and frequency domain graphs.

The first tab control is located on the left-hand side and offers three different selections. The first selection is default and is labelled as "Test Results." This tab, shown in Fig. 12(a) first displays the current cycle number and information about when the test was started. Under these two boxes, live voltage, current, and power flow levels are displayed to the user. Due to the fact that the charger used in this experiment requires manual voltage adjustment, the voltage reading flashes in red when it exceeds the charging limit. The voltage limit for this battery is addressed in the datasheet and recommends a charging voltage between $14.4 \mathrm{~V}$ and $14.8 \mathrm{~V}$ [15]. Below the live measurements are two columns which display comprehensive details over the charge and discharge cycles. Both the charge and discharge detail columns offer the user information over the time and capacity transferred in the current cycle as well as cumulatively over the entire test period. The user can also toggle between different units they wish these values to be displayed in. In Fig. 12(b), a battery test has reached its 35th cycle, thus it was selected to display the cumulative test times in days (d) rather than minutes as shown in the current cycle. To be consistent, all capacities were selected to display in Amp-hours (Ah). The final statistic displayed in this tab is the "Rest Time Remaining" and is shown below the charge and discharge details. This value was initially added while the software was in development to verify charge and discharge details. This value was initially added while the software was in development to verify that all rest times were being observed. During the development stage, rest periods were set to very short times making a quick wait before charge and discharge phases started. However, in the final program, it was useful to know how much of the wait time remained before equipment needed to be ready.

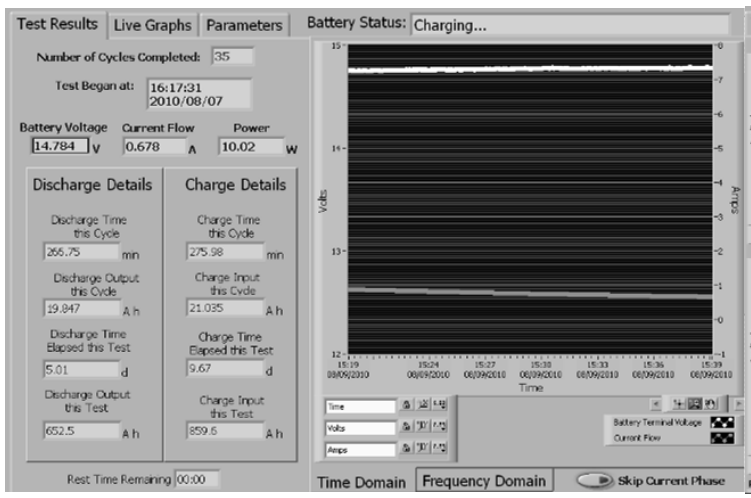

(a)

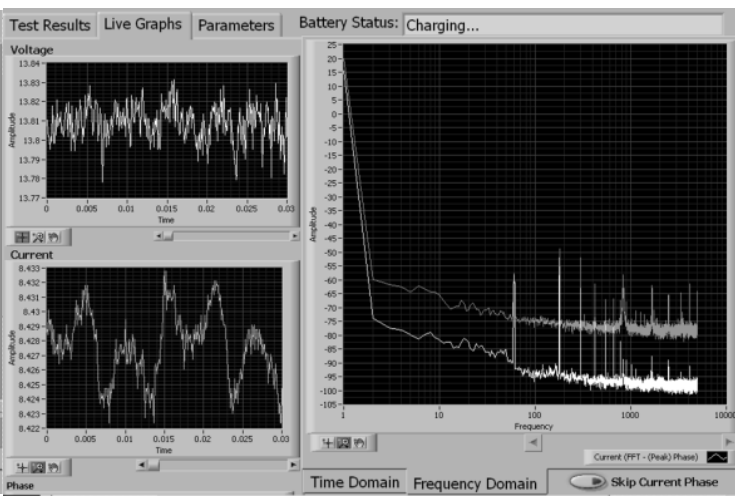

(b)

Fig. 12. Battery test stand program: (a) test results tab and (b) live graphs tab.

The second tab displays three individual "Live Graphs" to the user and is shown in Fig. 12(b). Although current and voltage are shown in the right tab control in Fig. 12(b), the window length is very long and is not able to display short time periods as well as small variations in magnitude. In the charge and discharge phases, small changes in magnitude are not particularly important and phases last for several hours thus a long time span is adequate. However, test pulses operate under a much shorter period and vary a great deal in magnitude. When viewing the current and voltage of these tests pulses in the 
right-hand tab the value appears flat and reveals no information about the pulse or its response. Shown in Fig. 12(b), the instantaneous voltage and current graphs are displayed to the left. In this example, the battery is in the charging phase and voltage and current plots are relatively constant only revealing the noise in the system. Fig. 12(b) also displays the DFT spectrum instead of the time domain representation but this can be easily toggled.

The final tab on the left-hand side labelled "Parameters" allows the user to review the test parameters that were initially set. Once a test has begun, the "Test Configuration" screen is no longer accessible. The "Parameters" tab provides a medium to review these values as well as check individual file locations where data is being saved. The second tab control located to the right of the test screen toggles the graph display. In the "Time Domain" tab, the battery voltage is shown in white, and current is shown in grey. Two vertical axes are displayed differentiating the battery voltage and current ranges. The left vertical axis displays the battery voltage and right displays the current flow. Several additional options are provided to adjust the graph position and range.

\section{Conclusion}

This paper presents and discusses the design of a battery testing system capable of fully charging and discharging a battery, providing insight into the improvement of battery SoC, voltage stability, and SoH. A laboratory testing system is constructed using a programmable load and controllable DC voltage supply connected to a LabVIEW data acquisition interface and control system. A comprehensive front-end GUI was built to analyze the battery in real-time as well as in the frequency domain with data logging capabilities. In addition to collecting real-time and historical data on battery performance during each cycle, three unique testing pulses were designed to analyze the battery response in the time and frequency domains. This system provides insight into the performance of a lead acid battery as well as the underlying electrochemistry by spectral analysis following the test pulses. This system can be used to generate performance and lifespan detail as well as provide an educational tool for classroom discussions in energy storage topics.

\section{References}

[1] Vincent CA. Modern Batteries: An Introduction to Electrochemical Power Sources. 2nd Ed. New York, NY USA: John Wiley \& Sons, Inc.; 1997:142-153.

[2] Berndt D. Maintenance-Free Batteries. Taunton, Somerset, England UK: Research Studies Press; 1997:97-127.

[3] Treptow RS. The lead-acid battery: its voltage in theory and in practice. J. Chem. Educ., Mar. 2002; 79(3):334.

[4] Bergvekd HJ, Kruijt WS, Notten PHL. Battery Management Systems: Design by Modeling. Boston, MA USA: Kluwer Academic Publishers; 2002; ch. 2.

[5] Piller M, Perrin AJ. Methods for state-of-charge determination and their applications. Journal of Power Sources, Jun. 2001; 96:113-120.

[6] Baronti F, Fantechi G, Fanucci L, Leonardi E, Roncella R, Saletti R, Saponara S. State-of-charge estimation enhancing of lithium batteries through a temperature-dependent cell model. In: Proc. the International Conference on Applied Electronics, 2011:1-5.

[7] Maxim. (Jun. 2003). DS2740 High-Precision Coulomb Counter Reference Design. Sunnyvale, CA USA. [Online]. Available: http://www.maxim-ic.com

[8] Patel MR. Spacecraft Power Systems. Boca Raton, FL USA: CRC Press; 2005:353.

[9] Chang WY. (2013). The state of charge estimating methods for battery: a review. ISRN Applied Mathematics. [Online]. Available: http://dx.doi.org/10.1155/2013/953792

[10] Malkhandi S. Fuzzy logic-based learning system and estimation state-of-charge of lead-acid battery. Engineering Applications of Artificial Intelligence, Aug. 2006; 19:479-485.

[11] Barsoukov E, Macdonald JR. Impedance Spectroscopy: Theory, Experiment, and Applications. Hoboken, NJ USA: John Wiley \& Sons, Inc.; 2005; ch. 1.

[12] Kozlowski JD. Electrochemical cell prognostics using online impedance measurements and model-based data fusion techniques. In: Proc. the IEEE Aerospace Conference, 2003; 7:3257-3270.

[13] Zou Y, et al. Combined state of charge and state of health estimation over lithium-ion battery cell cycle lifespan for electric 
vehicles. Journal of Power Sources, 2015; 273:798-803.

[14] Crompton TR. Battery Reference Book. Woburn, MA USA: Reed Educational \& Professional Publishing, Ltd.; 2001:31/331/4.

[15] Power Sonic. PS-12260 12 Volt 26.0 AH Rechargeable Sealed Lead Acid Battery. [Online]. Available: http://www.powersonic.com

[16] Lashway CR, Mohammed O. Improved estimation methods for lead acid utility arrays for microgrids. In: Proc. the IEEE Power Energy Society on Innovative Smart Grid Technologies Conference, 2015:1-5.

[17] HP. Regulated DC Power Supply Models 6256B, 6263B, 6264B, 6265B, 6266B, 6267B, 6271B, and 6274B. Palo Alto, CA USA: Hewlett-Packard Company; Jan. 1976.

[18] Amrel, Programming Manual PLA-PLW Series. El Monte, CA USA: American Reliance, Inc.; 2006.

[19] Coleman M, Hurley WG, Lee CK. An improved battery characterization method using a two-pulse load test. IEEE Transactions on Energy Conversion, Jun. 2008; 23:708-713.

[20] Das AD, Mahapatra KK. Real-Time implementation of Fast Fourier Transform (FFT) and finding the power spectrum using LabVIEW and CompactRIO. In: Proc. the International Conference on Communication Systems and Network Technologies, 2013. 